\title{
PROBABILISTIC ASPECTS OF HIGH-DYNAMIC COMPRESSIVE FAILURE OF CEMENTITIOUS MATERIALS
}

\author{
BERNHARD PICHLER* AND ERHARDT LACH ${ }^{\dagger}$ \\ *TU Wien - Vienna University of Technology \\ Vienna, Austria \\ e-mail: Bernhard.Pichler@tuwien.ac.at \\ ${ }^{\dagger}$ French-German Research Institute of Saint Louis (ISL) \\ Saint Louis, France \\ e-mail: elach@gmx.de
}

Key words: Dynamic strength increase factor, Cement paste, Mortar, Concrete

\begin{abstract}
When subjecting cementitious materials to high-dynamic uniaxial compression, the ultimate load carrying capacity of tested specimens increases significantly with increasing loading rate. In the present paper, an engineering mechanics model [6,7] which explains high-dynamic strengthening of cement pastes, mortars, and concretes under uniaxial compression is discussed. The model explains that high dynamic strengthening is probabilistic in the sense that the first crack may nucleate at any position within the volume of the tested specimen, and that the actual position of crack nucleation is relevant for the achievable compressive strength of the specimen. The disintegration process of the specimen, namely, starts with nucleation of the first crack and lasts until the crack has propagated - in loading direction - through the entire specimen. This process lasts the longer, the closer the first crack nucleates to one of the load platens. During the disintegration process, the stress level increases inside the solid parts of the specimens, driven by progressive macroscopic load increase. Crack nucleation is modeled by an elastic limit criterion. Crack propagation is considered to happen under mode I, in direction of uniaxial loading, at the speed of Rayleigh waves. The ultimate load is equal to the product of the elastic stress rate and the total test duration. The model does not include any fitting parameters. Still, model predictions agree qualitatively and quantitatively very satisfactorily with measured dynamic strength increase factors. This suggests that high-dynamic strengthening is a purely structural effect, depending on geometric properties of the tested specimen.
\end{abstract}

\section{INTRODUCTION}

When subjecting cementitious materials to high-dynamic uniaxial compression, the ultimate load carrying capacity of tested specimens increases significantly with increasing loading speed. It is also well known that experimentally determined high-dynamic "strength" values exhibit significantly more dispersion that their quasi-static counterparts. As regards explanations for these two effects, the scientific community is still debating about contributions from the visco-elasticity of the bulk material, from loading-rate dependent aspects of crack propagation, and from inertia forces, see, e.g., [1-5], but it remains unclear to which extents high-dynamic strengthening is a material effect or a structural effect, respectively.

The present paper reports on an engineering mechanics model which explains high-dynamic strengthening of cement pastes, mortars, and concretes under uniaxial compression [6, 7]. 
This model is free from fitting parameters. Given our focus on compressive strength testing, we consider compression to be positive and tension to be negative.

\section{HIGH DYNAMIC STRENGTH OF CE- MENTITIOUS MATERIALS}

In high-dynamic compression tests, the crushed specimens fall into many small pieces. From a-posteriori inspection of fracture planes of a cement paste sample, it was concluded in [6] that cracks propagate predominantly in the direction of uniaxial compressive loading. This implies a so-called "axial splitting" failure mode and has resulted in the following modeling idea [6].

Consider a high-dynamic uniaxial compressive strength test carried out at a given strain rate $\dot{\varepsilon}$. Consider also that time $t$ starts to run, once loading of a specimen begins. Consequently, the total strain follows at any time $t$ as

$$
\varepsilon(t)=t \cdot \dot{\varepsilon} .
$$

Consider that a high-dynamic strength test is performed so fast that its duration is so short that creep cannot contribute significantly to the total deformations of the tested specimens. Therefore, specimens behave linear-elastically and Young's modulus $E$ is the proportionality factor between stresses and strains

$$
\sigma(t)=E \varepsilon(t) \text {. }
$$

As for more detailed modeling, we subdivide a high-dynamic strength test into three consecutive phases, discussed next.

\subsection{First phase of a high-dynamic compres- sion test}

During the first phase of a high-dynamic strength test, the specimen remains intact. The phase ends, as soon as the first macrocrack nucleates. Consider that this will be the case, once the stress in the high-dynamic test has reached the static compressive strength $f_{c u}$ of the material. Consequently, the stress level at the end of the first phase, i.e. at time $t=\Delta t_{1}$, reads as

$$
\sigma\left(\Delta t_{1}\right)=f_{c u} .
$$

\subsection{Second phase of a high-dynamic com- pression test}

During the second phase of a high-dynamic strength test, the first macrocrack propagates in loading direction. The phase ends, as soon as the crack has propagated through the entire specimen, such that the crack has split the specimen into two halves. Let us quantify the incremental stress increase during the second phase. This requires knowledge (i) on the speed of crack propagate and (ii) on the position at which the crack has nucleated, as discussed next.

As for the speed of crack propagation $v_{\text {crack }}$, it is noteworthy that nanoscopic calciumsilicate-hydrates are the microstructural glue of cementitious materials. There exist many interfaces between different calcium-silicatehydrates. It is very likely, that cracks will propagate along such interfaces. Notably, molecular dynamics simulations of mode I crack propagation along material interfaces have shown (i) that cracks accelerate very quickly to the Rayleigh wave speed, and (ii) that crack propagation is limited by this speed [8]. Therefore, we consider that the speed of crack propagation is constantly equal to the Rayleigh wave speed. The latter is very similar to the speed of normal shear waves [8, 9], also referred to as transversal waves. Their speed is equal to the square root of the ratio between shear modulus $G$ and mass density $\varrho$ of the material, i.e. $v_{\text {shearwave }}=\sqrt{G / \varrho}$. Combining the given arguments, the crack propagation speed is estimated as

$$
v_{\text {crack }}=\sqrt{G / \rho} .
$$

The time which is required such that the first crack splits the sample, depends on the position at which the first crack nucleates. In this context, let $d$ denote the geometric size of the specimen in loading direction. All real cases are bounded by the following two extreme cases. (i) If the first crack nucleates right at the center of the specimen, two opposite crack edges will propagate in loading direction. The specimen will be split into two halves, once each of the two crack edges has propagated along half 
of the specimen size. Therefore, the minimum duration of the second phase of a high-dynamic compression tests follows under consideration of crack propagation speed (4) as

$$
\min \Delta t_{2}=0.5 d \sqrt{\rho / G}
$$

(ii) If the first crack nucleates in the immediate vicinity of one of the load platens, only one crack edge will propagate in loading direction. The specimen will be split into two halves, once this active edge has propagated along the full size of the specimen $d$. Therefore, the maximum duration of the second phase of a highdynamic compression tests follows under consideration of crack propagation speed (4) as

$$
\max \Delta t_{2}=d \sqrt{\rho / G}
$$

This introduces a probabilistic nature of highdynamic strength tests. Since any position between the two load platens is a sound candidate for nucleation of the first crack, one has to consider an interval of possible durations for the second phase of a high-dynamic compression tests. This interval ranges between the two calculated extreme values:

$$
\Delta t_{2}=[0.5,1.0] d \sqrt{\rho / G}
$$

The material behavior during the second phase of a high-dynamic strength tests is discussed next. While the first crack propagates in loading direction, the material beside the crack remains intact. Therefore, it will continue to behave linear elastically. Since the loading of the specimen continues, also the stress in the solid volume of the specimen increases accordingly. Progressive increase of the stress level makes it very likely that additional cracks will nucleate at other positions, resulting in multiple crack propagation. While this explains why a specimen falls into many small pieces during a highdynamic strength test, let us consider that all cracks propagate in loading direction and that the material between the cracks remains linear elastic, with further increasing stress.

The stress level reached at the end of the second phase of a high-dynamic strength test is quantified next. The related strain increment follows from combination of Eq. (1) with (7) as $\Delta \varepsilon\left(\Delta t_{2}\right)=[0.5,1.0] d \sqrt{\rho / G} \cdot \dot{\varepsilon}$. Inserting this strain increment into the linear elastic law (2) delivers the corresponding stress increment as $\Delta \sigma\left(\Delta t_{2}\right)=[0.5,1.0] d E \sqrt{\rho / G} \cdot \dot{\varepsilon}$.

\subsection{Third phase of a high-dynamic com- pression test}

Consider that the transition from the second to the third phase marks the peak load in a high dynamic strength tests. Consequently, the stress inside the specimen decreases during the third phase of a high-dynamic compression tests, because the specimen falls into pieces. This implies that the ultimate stress during a high-dynamic compressive strength test is equal to the stress level reached at the end of the second phase. This ultimate stress level is equal to the sum of (i) the stress level $f_{c u}$ reached at the end of the first phase, and (ii) the stress increment of the second phase:

$$
\sigma_{u l t}(\dot{\varepsilon})=f_{c u}+[0.5,1.0] d E \sqrt{\rho / G} \cdot \dot{\varepsilon}
$$

\subsection{Dynamic strength increase factor}

The dynamic strength increase factor, DIF, is the ratio between a high-dynamic strength values $\sigma_{u l t}(\dot{\varepsilon})$, see (8), and the quasi-static strength $f_{c u}$, i.e.

$$
\operatorname{DIF}(\dot{\varepsilon})=1+[0.5,1.0] \frac{d E}{f_{c u}} \sqrt{\frac{\rho}{G}} \cdot \dot{\varepsilon}
$$

This expression for the dynamic strength increase factor contains - as required input values - just material properties and the specimen size measured in loading direction. These inputs are of clear physical meaning and simple to quantify. Therefore, we now strive for model validation.

\section{Model validation}

In the sequel, we evaluate the dynamic strength increase factor, DIF, according to Eq. (9), and we compare model outputs with experimental data. To this end, we consider highdynamic uniaxial compressive strength tests 
carried out on cement paste, mortar, and concrete.

\subsection{Cement paste}

As for cement paste, we consider the quasi-static and high-dynamic tests described in Fischer et al. [6]. The input quantities for the DIF expression (9) are listed in Table 11. Model predictions agree very well with the tested strength values, see Fig. 1

Table 1: Cement paste properties from Fischer et al. [6]

\begin{tabular}{lc}
\hline specimen height & $d=6.625 \mathrm{~mm}$ \\
\hline Young's modulus & $E=14.24 \mathrm{GPa}$ \\
\hline shear modulus & $G=5.533 \mathrm{GPa}$ \\
\hline mass density & $\varrho=1597 \mathrm{~kg} / \mathrm{m}^{3}$ \\
\hline static strength & $f_{c u}=48.15 \mathrm{MPa}$ \\
\hline
\end{tabular}

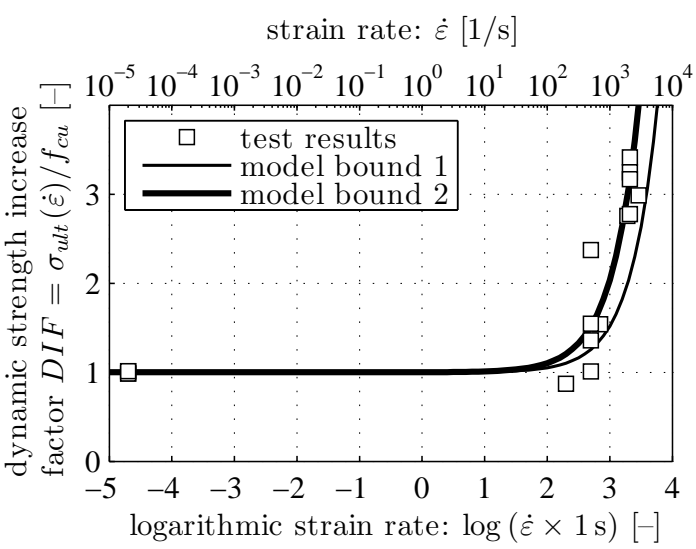

Figure 1: Comparison of (i) model predictions obtained from specialization of DIF expression (9) for input quantities listed in Table 1 and (ii) experimental data from [6]; after [6]

\subsection{Mortar}

As for mortar, we consider the quasi-static and high-dynamic tests described in Grote et al. [10]. The input quantities for the DIF expression (9) are listed in Table 2. Model predictions agree very well with the tested strength values, see Fig. 2,

\subsection{Concrete}

As for concrete, we consider the quasi-static and high-dynamic tests described in Gary and Bailly [11]. The
Table 2: Mortar properties from Grote et al. [10]

\begin{tabular}{ll}
\hline specimen height & $d=1.524 \mathrm{~cm}$ \\
\hline Young's modulus & $E=20 \mathrm{GPa}$ \\
\hline shear modulus & $G=8.3 \mathrm{GPa}$ \\
\hline mass density & $\varrho=2100 \mathrm{~kg} / \mathrm{m}^{3}$ \\
\hline static strength & $f_{c u}=80 \mathrm{MPa}$ \\
\hline
\end{tabular}

strain rate: $\dot{\varepsilon}[1 / \mathrm{s}]$

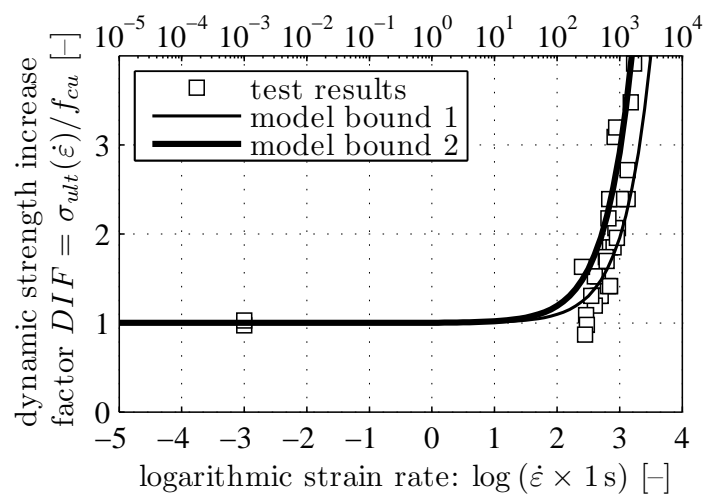

Figure 2: Comparison of (i) model predictions obtained from specialization of DIF expression (9) for input quantities listed in Table 2 and (ii) experimental data from [10]; after [6]

model remains applicable, if $d$ denotes the maximum aggregate size. The underlying idea is that once the first crack has extended to a length being equal to the maximum grain size, a large aggregate will break out of the microstructure of the specimen, and this marks the peak load in a high-dynamic uniaxial compression test. The input quantities for the DIF expression (9) are listed in Table 3. Model predictions agree very well with the tested strength values, see Fig. 3 .

\section{CONCLUSIONS}

The presented engineering mechanics model does not contain any fitting parameter and explains the high-dynamic compressive strength increase of cement paste, mortar, and concrete reliably. The model suggests that crack nucleation starts - independent from the loading rate - as soon as the quasistatic strength is reached. In a quasi-static test, the loading rate is so slow that crack- 
Table 3: Concrete properties from Gary \& Bailly [11]

\begin{tabular}{ll}
\hline maximum grain diameter & $d=1 \mathrm{~cm}$ \\
\hline Young's modulus & $E=32 \mathrm{GPa}$ \\
\hline shear modulus & $G=13.3 \mathrm{GPa}$ \\
\hline mass density & $\varrho=2300 \mathrm{~kg} / \mathrm{m}^{3}$ \\
\hline static strength & $f_{c u}=48 \mathrm{MPa}$ \\
\hline
\end{tabular}

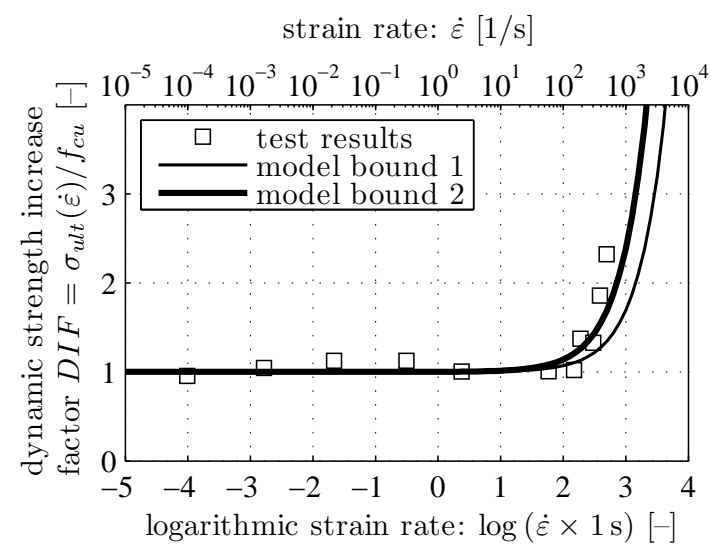

Figure 3: Comparison of (i) model predictions obtained from specialization of DIF expression (9) for input quantities listed in Table 3 and (ii) experimental data from [11]; after [7]

ing is a quasi-instantaneous process. In other words, the interval of time which is required to load a specimen up to the quasi-static strength is significantly larger than the interval of time which is required for the first crack to propagate though the entire sample and to split it into two halves. In a high-dynamic test, in turn, the loading rate is so fast that consideration of a finite speed of crack propagation is important. In other words, the interval of time which is required to load a specimen up to the quasi-static strength is of similar size or smaller than the interval of time which is required for the first crack to propagate though the entire sample and to split it into two halves. Therefore, a significant increase of the stress in the solid parts of the specimen is possible during its disintegration process. This suggests that high-dynamic increase of uniaxial compressive strength is a structural effect, because it involves the geometric dimension of the specimen, measured in the direction of loading.

The model provides interesting insight into the probabilistic nature of high-dynamic strength tests. The significant dispersion of high-dynamic strength values results from uncertainty regarding the position at which the first crack nucleates. If the first crack nucleates at the center of the specimen, two opposite crack edges are active. In order to split the specimen, both of them have to run just along half of specimen size in loading direction. This will result in the shortest duration and the smallest high-dynamic strength value. If the first crack nucleates directly at one of the load platens, just one crack edges is active. In order to split the specimen, it has to run along the total specimen size in loading direction. This will result in the longest duration and the largest high-dynamic strength value. Realistic scenarios will fall in between these extreme cases. This explains the significant dispersion observed in high-dynamic strength testing.

\section{REFERENCES}

[1] P.H. Bischoff and S.H. Perry. Compressive behaviour of concrete at high strain rates. Materials and Structures, 24(6):425-450, 1991.

[2] M. Zhang, H.J. Wu, Q.M. Li, and F.L. Huang. Further investigation on the dynamic compressive strength enhancement of concrete-like materials based on split Hopkinson pressure bar tests. Part I: Experiments. International Journal of Impact Engineering, 36(12):1327-1334, 2009.

[3] Z. P. Bažant and Y.-N. Li. Cohesive crack with rate-dependent opening and viscoelasticity: I. mathematical model and scaling. International Journal of Fracture, 86(3):247-265, 1997.

[4] Q.M. Li and H. Meng. About the dynamic strength enhancement of concretelike materials in a split Hopkinson pressure bar test. International Journal 
of Solids and Structures, 40(2):343-360, 2003.

[5] X.Q. Zhou and H. Hao. Modelling of compressive behaviour of concrete-like materials at high strain rate. International Journal of Solids and Structures, 45(17):46484661, 2008.

[6] I. Fischer, B. Pichler, E. Lach, C. Terner, E. Barraud, and F. Britz. Compressive strength of cement paste as a function of loading rate: Experiments and engineering mechanics analysis. Cement and Concrete Research, 58:186-200, 2014.

[7] B. Pichler, I. Fischer, E. Lach, C. Terner, E. Barraud, and F. Britz. The influence of loading rate on the compressive strength of cementitious materials: experiments and separation of time scales-based analysis. In Computational Modelling of Con- crete StructuresProceedings of EURO-C 2014, volume 2, pages 731-773. 2014.

[8] F.F. Abraham and H. Gao. How fast can cracks propagate? Physical Review Letters, 84(14):3113-3116, 2000.

[9] I. A. Viktorov. Rayleigh and Lamb waves: Physical theory and applications. Plenum Press (New York), 1967.

[10] D.L. Grote, S.W. Park, and M. Zhou. Dynamic behavior of concrete at high strain rates and pressures: I. Experimental characterization. International Journal of Impact Engineering, 25(9):869-886, 2001.

[11] G. Gary and P. Bailly. Behaviour of quasibrittle material at high strain rate. Experiment and modelling. European Journal of Mechanics, A/Solids, 17(3):403-420, 1998. 